\title{
Resiliencia de los adultos mayores del Centro Diurno Gerontológico "Cristo Rey" frente al abandono de sus familiares
}

Resilience of the elderly of the "Cristo Rey" Gerontological Day Center against the abandonment of their relatives

Vicenta Inmaculada Ramona Aveiga Macay

Evelyn Lilibeth Cedeño Aguayo2

RECIBIDO: 9 DE ABRIL DE 2020

ACEPTADO: 5 DE JUNIO DE 2020

\section{RESUMEN}

El objetivo de este estudio fue demostrar la resiliencia de los adultos mayores del Centro Diurno Gerontológico "Cristo Rey” frente al abandono de sus familiares. Para realizar el proceso de investigación se desarrolló una revisión bibliográfica de diversos artículos científicos, de los cuales se obtuvo información relevante sobre la resiliencia en la vejez. La investigación descriptiva permitió explicar la realidad de los adultos

\footnotetext{
1 PhD en Ciencias Pedagógicas, Magíster en Gerencia Educativa Profesional, Docente Investigadora, Universidad Técnica de Manabí, Ecuador; vaveiga@utm.edu.ec,

ORCID: https://orcid.org/0000-0001-8028-7432

Google Scholar: https://scholar.google.es/citations?user=lhoEyJYAAAAJ\&hl=es

2 Egresada de Trabajo Social, Universidad Técnica de Manabí, Ecuador; ecedeno3011@utm.edu.ec evelyncedeno97@gmail.com, ORCID: https://orcid.org/0000-0002-9147-2657

Google Scholar: https://scholar.google.es/citations?hl=es\&user=KDLTJg0AAAAJ
} 


\section{0}

mayores de la institución. Se utilizó el enfoque cuali-cuantitativo mediante el análisis de las técnicas de la entrevista realizada a veinte adultos mayores que asisten diariamente al centro y un cuestionario de seis interrogantes a cincuenta adultos mayores que se encuentran registrados en el mismo. Este instrumento fue estructurado por las autoras como resultado de la interpretación de estudios realizados sobre la resiliencia y el análisis de la conjunción entre el modelo de las verbalizaciones de Grotberg (yo soy, yo estoy, yo tengo, yo puedo) y la Escala de Resiliencia desarrollada por Eugenio Saavedra y Marco Villalta en el año 2008, en donde los resultados obtenidos pudieron evidenciar que en el proceso del envejecimiento, el adulto mayor en la ciudad de Portoviejo se muestra afectado de manera emocional, física y mentalmente por motivo del abandono de sus familiares. Por ende, la labor del trabajador social del Centro Diurno "Gerontológico Cristo Rey" es conocer y transformar la realidad del mismo, de tal modo que es necesario poseer la mayor cantidad de datos para realizar un buen diagnóstico y orientación que permita dar respuesta a las necesidades de acuerdo a los recursos disponibles y de esta manera promover un envejecimiento activo.

Palabras clave: resiliencia, adulto mayor, abandono, envejecimiento activo

\section{ABSTRACT}

The objective of this study was to demonstrate the resilience of older adults at the "Cristo Rey" Gerontological Day Center against abandonment by their relatives. To carry out the research process, a bibliographic review of several scientific articles was developed from which relevant information was obtained. on resilience in old age. The descriptive research allowed to explain the reality of the older adults of the institution. The qualitative-quantitative approach was used by analyzing the techniques of the interview carried out with twenty older adults who attend the center daily and a questionnaire of six questions to fifty older adults who are registered in it, where the results obtained could show that in the aging process the elderly in the city of Portoviejo is emotionally, physically and mentally affected due to the abandonment of their relatives, therefore the work of the social worker of the Day Center "Gerontológico Cristo Rey" is to know and transform the reality of the same in such a way that it is necessary to possess the greatest amount of data through to carry 
out a good diagnosis and orientation that allows to respond to the needs according to the available resources and thus promote active aging.

Keywords: resilience, older adult, neglect, active aging

\section{Introducción}

Una realidad importante en la sociedad son los adultos mayores. Ellos han prolongado su existencia entregando sus conocimientos y sabiduría, orientando y formando una familia. Con su experiencia, carácter y fuerza de voluntad, han logrado posesionarse en un contexto que contemplan con agrado, así como las huellas que han marcado sus caminos.

En este sentido, Cárdenas y López (2011), expresan que la vejez es un período de retos adaptativos, dado el cambio gradual de las condiciones de salud física y mental, las dificultades para el desarrollo de las actividades de la vida diaria y las restricciones a la participación social. Es así que cuando se llega a esta etapa de la vida, la persona ha logrado acumular una serie de conocimientos, actitudes y experiencias para afrontar situaciones de cotidianidad y adversidad.

Con el paso de los años también se agudizan las dolencias físicas, el cuerpo ya no es el mismo de antes y se requiere de una atención especializada. El abandono de los familiares provoca depresión, estado de ansiedad, nerviosismo, aislamiento e inapetencia, afectando el estado físico y mental en los adultos mayores. En este contexto de soledad, se vuelven indefensos y vulnerables para afrontar necesidades básicas de dependencia, intimidad y relaciones con otros.

Como resultado de este estado emocional, el adulto mayor asume actitudes como abandonar de sí mismo y perder motivación ante la vida, de tal modo que la resiliencia juega un papel importante en el proceso del envejecimiento, por ser la habilidad que posee el ser humano para adaptarse de manera positiva a situaciones adversas. En este sentido, se resulta oportuno lo expuesto por Garmezy (2006), cuando define a la resiliencia como "la capacidad para recuperarse y mantener una conducta adaptativa después del abandono o la incapacidad para iniciar un evento estresante". 


\section{2}

En este contexto, la Organización Mundial de la Salud (2002), considera que el envejecimiento activo es "el proceso de optimizar las oportunidades de salud, participación y seguridad con el objetivo de mejorar la calidad de vida de las personas a medida que envejecen".

La Constitución de la República de Ecuador (2008) tipifica en sus artículos que estos lineamientos son competencias del Estado, ante lo cual ha implementado programas en beneficio de este grupo de atención prioritaria, promoviendo el desarrollo de nuevas estrategias para cubrir necesidades básicas: salud, trabajo, cuidados, protección social y convivencia intergeneracional.

Sobre la base de las consideraciones anteriores, en la ciudad de Portoviejo, provincia de Manabí, se encuentra el Centro Diurno Gerontológico "Cristo Rey", institución no gubernamental y sin fines de lucro que tiene el propósito de conocer la situación de salud y social de los adultos mayores de la zona que presentan bajos recursos económicos, discapacidades e indigentes, para brindarles atención oportuna mediante proyectos que la institución oferta basada en sus políticas internas. El objetivo de esta investigación es demostrar la resiliencia de los adultos mayores del Centro Diurno Gerontológico "Cristo Rey" de la ciudad de Portoviejo frente al abandono de sus familiares.

\section{Desarrollo}

En el siglo XXI se considera que el envejecimiento de la población mundial es un fenómeno que marcará estos tiempos debido a que cada segundo dos personas cumplen sesenta años y hasta la actualidad existen ochocientas diez millones de personas mayores a esa edad en el mundo. De acuerdo a las Estadísticas Sanitarias Mundiales del 2009 de la Organización Mundial de la salud, las personas mayores de sesenta años representan el $11 \%$ de la población total, por lo que se requiere que la atención de este grupo se focalice en promover la salud, prevenir enfermedades y garantizar un envejecimiento adecuado, que se ve influenciado por la capacidad de resiliencia de las personas, las cuales van superando adversidades que se presentan durante el transcurso de su vida. En este sentido, las personas más resilientes tienen más armas para poder enfrentarlas y no verse sometidos a desequilibrios emocionales. 
Resulta oportuno destacar que el Instituto Nacional de Estadísticas y Censos (2016), establece que en Ecuador las cifras demuestran que los adultos mayores representan el 25\% de la población nacional. Este grupo de personas deberían ser prioridad para los estadistas y planificadores en la creación de modelos de desarrollo, por cuanto la creciente práctica del abandono de la familia y desprotección hacia ellos puede provocar consecuencias como la soledad, esto representa un estado de aislamiento donde la persona se vuelve vulnerable e indefensa para afrontar necesidades básicas de dependencia, intimidad y relación con otros. Este hecho se debe a que los hijos se van del hogar para conformar nuevas familias y las visitas se vuelven esporádicas. Otra de las razones es por viudez, ante la partida de la pareja de toda la vida y la más lamentable es por abandono de la propia familia. A pesar de ello, los adultos mayores se han acoplado y sobrevivido a un sinnúmero de situaciones valiéndose de procesos de adaptación, mediante los cuales logran mantener un adecuado nivel de funcionamiento en diferentes dominios $y$, felizmente, alta o adecuada satisfacción con la vida y bienestar; es decir, han mostrado resiliencia ante las adversidades (Brandtstädter y Greve, 1994).

Alentar a las personas mayores a desarrollar su potencial de independencia es una intervención que puede extender los años de vida, prevenir hospitalizaciones, evitar enfermedades y lesiones causadas por la inmovilidad y el estilo de vida sedentario (Machado, 2010). En consideración de este análisis, Jiménez (2011), manifiesta que a pesar de que el adulto mayor pueda presentar dificultades durante el proceso de envejecimiento, es preciso trabajar en su resiliencia, de modo que se ha visto que las persona mayores tienden a prestar más atención a los estímulos positivos que a los negativos, favoreciendo la adaptación ante los acontecimientos vitales diversos por los que puede pasar una persona.

Lascano (2017), manifiesta que "Ios ancianos en esta etapa de su vida, se enfrentan no sólo a cambios físicos o a cambios que involucran un deterioro cognitivo; sino que también es probable que manifiesten ciertos sentimientos de abandono o soledad que podrían ser causados a raíz de que algunas veces sus familiares no los comprenden y más bien los toman como si fueran una carga". Un grupo de ancianos ingresa a un centro de día por voluntad propia y otro grupo considerable lo hace porque sus familiares los inscribieron en esos lugares. Si en dichos centros no se les 


\section{4}

proporciona un ambiente adecuado y una atención oportuna, el adulto mayor podría reflejar sentimientos de depresión, angustia y ansiedad, llegando incluso algunas veces a presentar psicopatologías tales como psicosis o neurosis agudas (Organización Mundial de la Salud, 2015).

Hoy en día, el adulto mayor está respaldado por la Constitución de la República del Ecuador (2008), destacando el artículo №36 el cual tipifica que las personas adultas mayores deben recibir atención prioritaria y especializada tanto en el ámbito público como privado, en especial en los campos de inclusión social y económica, y protección contra la violencia. Las personas adultas mayores son aquellas que hayan cumplido los sesenta y cinco años de edad. Además, el artículo $N^{\circ} 37$ indica que el adulto mayor debe tener una acertada asistencia económica y psicológica para garantizar su estabilidad mental y física. Para tal efecto, se sancionará a los familiares que hayan abandonado a los adultos mayores.

El Ministerio de Inclusión Económica y Social (MIES), fomenta el envejecimiento activo para tener una vida saludable y digna, por medio de los centros de atención directa, entre ellos el Centro Diurno Gerontológico Cristo Rey, ubicado en la Provincia de Manabí en la ciudad de Portoviejo. En su gran mayoría, las personas adultas mayores de la ciudad no tienen conocimiento sobre los programas que proporciona el Estado. Debido a esto, los trabajadores sociales deben realizar visitas domiciliarias a las comunidades más vulnerables para brindar atención a las necesidades de salud, cuidados, protección social y convivencia que les permitan tener una vejez fructífera.

En el Centro Diurno Gerontológico Cristo Rey se reciben a los adultos mayores de bajos recursos económicos, discapacitados e indigentes que solo requieren de un lugar en donde pasar el día y sentirse acogidos. Actualmente, se encuentran registrados setenta adultos mayores, de los cuales a diario asisten entre veinte y veinticinco personas. Esta institución no gubernamental, sin fines de lucro, ha logrado una presencia importante en la ciudad de Portoviejo. Inició sus actividades un 17 de Julio del año 2000, brindando servicio social dirigido a ubicar y resolver las necesidades más sentidas de la población, en especial de los sectores más vulnerables, entre ellos los adultos mayores. Tiene como misión brindar asistencia social el más alto nivel de calidad y calidez, contribuyendo al mejoramiento de 
calidad de vida a través de sus proyectos que se han venido desarrollando con éxito al pasar de los años: "El Comedor Padre Alonso Ascanio Mendoza S.J", "Centro Médico Cristo Rey" y el "Centro de rehabilitación física".

El objetivo de la intervención gerontológica del trabajador social en el "Centro Gerontológico Cristo Rey" es conocer y transformar la realidad del adulto mayor, colaborando con profesionales como psicólogos, terapistas físicos, terapistas ocupacionales, médicos y enfermeros para alcanzar el bienestar colectivo, entendido como un sistema global de acciones, que eleva el desarrollo del bienestar humano en los procesos de cambio social, tanto individual como familiar y/o comunitario (Cárdenas y Cedeño, 2018).

El Trabajo Social es una profesión que propone cambios, presenta propuesta a los conflictos de la sociedad, resume el desafío de interpretar los retos de grupos sociales, a fin de ubicarlos en nuestro tiempo. Esto quiere decir que es un profesional calificado, que refuerza y amplía su capacidad crítica, no solamente a nivel operativo, sino que también reflexiona, piensa, analiza, investiga, descifra la realidad y contribuye al mejoramiento de las condiciones (Ramos, Gonzales y Pacheco, 2015). El centro diurno brinda servicios multimodales; es decir; diurno, atención domiciliaria, espacios activos de socialización y encuentro en donde la trabajadora social es responsable de brindar cuidado a las setenta personas adultas mayores en todas las modalidades, la misma que realiza una evaluación preliminar de los adultos mayores que solicitan su ingreso al centro a través de la aplicación de las fichas sociales, valora la demanda y crea un diseño la intervención individual integral diario en función a las necesidades existentes y de los recursos disponibles, puesto que la interacción del adulto mayor con el medio y los vínculos extras mejoran su calidad de vida y supervivencia.

En esta labor se debe participar junto al equipo multidisciplinario para realizar la evaluación de la persona adulta mayor, de igual manera con la organización, programación y evaluación de las diversas actividades del centro diurno; además contribuir en la readaptación y rehabilitación psíquica y física que se logra a través de la estimulación cognitiva, motricidad fina, ludoterapia, aeróbicos, ejercicios, actividades cognitivas, musicoterapia, bailoterapia y jardinería como medio de conexión con el ecosistema. El trabajador social procura la reinserción del adulto 


\section{6}

mayor a su núcleo familiar mediante talleres para con la familia, realiza visitas domiciliarias como parte del seguimiento del caso y emite el informe social con las debidas recomendaciones.

De esa manera, el trabajo social parte del aprovechamiento de las fortalezas y oportunidades que ofrece la resiliencia. En ese sentido, el enfoque se perfila en torno a lo que funciona bien, a los factores que hacen que el adulto mayor se oriente a la búsqueda de alternativas de crecimiento social, pese a las condiciones sociales de adversidad. Esto podría permitir a la profesión, ensayar caminos que le amplíen sus perspectivas de cómo las personas afrontan la vida cotidiana en forma única y exclusiva, partiendo de sus potencialidades (Casas y Campos, 2008).

\section{Método}

El estudio se realizó en el "Centro Diurno Gerontológico Cristo Rey" ubicado en la ciudadela Pacheco, de la ciudad de Portoviejo - Manabí - Ecuador. La investigación realizada fue de tipo descriptiva, por cuanto permitió explicar la realidad de este grupo de atención prioritaria en el centro gerontológico; además se desarrolló una revisión bibliográfica de diversos artículos científicos con los cuales se obtuvo información relevante sobre la resiliencia del adulto mayor.

Para alcanzar el objetivo de este estudio se utilizó el enfoque cuali-cuantitativo, mediante el análisis de la entrevista realizada a veinte adultos mayores que asisten diariamente al centro gerontológico y un cuestionario de seis interrogantes a cincuenta adultos mayores que se encuentran registrados en el mismo. Este instrumento es resultado de la interpretación de estudios realizados sobre la resiliencia, mediante los cuales se identifica que la diversidad de las actividades, romper la rutina, hacer amigos, permanecer en compañía; pero también, realizar intervenciones profesionales a fin de que los adultos mayores permanezcan activos, nos muestra, de algún modo, el aprendizaje de la resiliencia y la incorporación de la misma. Además, se complementó este estudio con un análisis de la conjunción entre el modelo de las verbalizaciones de Grotberg (yo soy, yo estoy, yo tengo, yo puedo) y la Escala de Resiliencia desarrollada por Eugenio Saavedra y Marco Villalta en el año 2008, que señala la existencia de cuatro ámbitos de profundidad (Condiciones 
de base, visión de sí mismo, visión del problema y respuesta resilientes). Como resultado del análisis e interpretación de lo descrito, las autoras estructuraron un cuestionario conformado por 6 interrogantes que permite identificar factores específicos que forman parte de la resiliencia de los adultos mayores. A continuación, se establecen los factores en estudio que respaldan las interrogantes planteadas en el cuestionario:

Interrogante $n^{\circ} 1$ - AUTONOMÍA (F2): juicios referidos al vínculo que el adulto mayor establece consigo mismo para definir su aporte particular a su estilo sociocultural.

Interrogante $\mathbf{n}^{\circ} 2$ - VínCULOS (F5): juicios referidos al valor de la socialización primaria y redes sociales con raíces en la historia personal.

Interrogante $n^{\circ} 3$ - GENERATIVIDAD (F12): juicios referidos a la posibilidad de pedir ayuda a otros para solucionar situaciones problemáticas.

Interrogante $n^{\circ} 4$ - REDES (F6): juicios referidos al vínculo afectivo que establece el adulto mayor con su entorno social cercano.

Interrogantes $n^{\circ} 5$ y $n^{\circ}$ 6.- MODELOS (F7): juicios referidos a la convicción del papel de las redes sociales cercanas para apoyar la superación de situaciones problemáticas nuevas.

\section{Tabla 1}

Ámbitos de profundidad

\begin{tabular}{|c|c|c|c|c|}
\hline & $\begin{array}{c}\text { Condiciones de } \\
\text { base }\end{array}$ & $\begin{array}{l}\text { Visión de sí } \\
\text { mismo }\end{array}$ & $\begin{array}{l}\text { Visión del } \\
\text { problema }\end{array}$ & $\begin{array}{l}\text { Respuesta } \\
\text { resiliente }\end{array}$ \\
\hline Yo soy, yo estoy & & F2 & & \\
\hline Yo tengo & F5 & F6 & F7 & \\
\hline Yo puedo & & & & $\mathrm{F} 12$ \\
\hline
\end{tabular}

\section{Resultados}




\section{8}

Para la obtención de los resultados se aplicó una entrevista a veinte adultos mayores que asisten diariamente al Centro Diurno Gerontológico Cristo Rey, y una encuesta a cincuenta adultos mayores que se encuentran inscritos en este centro por motivo de abandono. El objetivo fue demostrar la resiliencia en la institución. La encuesta se describe a continuación:

\section{Tabla 2}

¿En que ocupa su mayor parte del tiempo?

\begin{tabular}{l|ccc}
\hline & Asistir al centro & $\begin{array}{c}\text { Quedarse en } \\
\text { casa }\end{array}$ & Dormir \\
\hline Porcentaje & $96 \%$ & $3 \%$ & $1 \%$ \\
& & Total 100\% & \\
\hline
\end{tabular}

El 96\% de los adultos mayores del centro ocupan mayor parte de su tiempo en asistir al mismo, otro $3 \%$ no frecuenta acudir al centro diariamente prefiriendo quedarse en casa y el $1 \%$ prefiere dormir. Por dichos resultados, se puede concluir que el adulto mayor tiene la necesidad de acudir a un lugar en donde poder pasar el día acompañado de otras personas.

\section{Tabla 3}

¿Con quién vive?

\begin{tabular}{c|ccc}
\hline & Solo & Con un hijo & Otro \\
\hline Porcentaje & $94 \%$ & $4 \%$ & $2 \%$ \\
& & Total 100\% & \\
\hline
\end{tabular}

Al preguntar a los adultos mayores con quiénes viven, las respuestas fueron: El $94 \%$ vive solo, el $4 \%$ de ellos viven con un hijo y el $2 \%$ restante vive con otro familiar, siendo estos nieto/a, sobrino/a o hermano/a. Es un claro ejemplo de que la mayoría de sus familiares han abandonado a los adultos mayores.

\section{Tabla 4}

¿Qué le ayuda a superar situaciones difíciles? 


\begin{tabular}{l|ccc}
\hline & $\begin{array}{c}\text { Intervención del } \\
\text { Trabajador } \\
\text { Social }\end{array}$ & $\begin{array}{c}\text { Relacionarse } \\
\text { con el entorno }\end{array}$ & Ser optimista \\
\hline Porcentaje & $65 \%$ & $30 \%$ & $5 \%$ \\
& & Total 100\% & \\
\hline
\end{tabular}

La respuesta de los adultos mayores respecto a la interrogante de qué les ayuda a superar situaciones difíciles fueron: Al 65\% de ellos les ayuda el trabajador social mediante su intervención, el 30\% prefiere relacionarse con el entorno y a un $5 \%$ le ayuda el ser una persona optimista. Por lo que se concluye que la intervención del trabajador social en el centro es de gran importancia debido a su empeño por lograr un envejecimiento activo.

\section{Tabla 5}

¿Por qué considera necesario acudir al centro diariamente?

\begin{tabular}{c|ccc}
\hline & Por distracción & $\begin{array}{c}\text { Para evitar la } \\
\text { soledad }\end{array}$ & Para no deprimirse \\
\hline Porcentaje & $42 \%$ & $37 \%$ & $21 \%$ \\
& & Total $100 \%$ & \\
\hline
\end{tabular}

Ante la incógnita presentada se obtuvieron respuestas variadas puesto que, un $42 \%$ de los adultos mayores acude al centro por distracción, otro $37 \%$ indicaron que asisten para no deprimirse y en un $21 \%$ para evitar la soledad en la que viven. Se puede concluir que en su mayoría, los adultos mayores ya han logrado superar el abandono de sus familiares.

\section{Tabla 6}

¿En qué aspectos le beneficia la intervención del Trabajador Social del centro? 


\begin{tabular}{c|ccc}
\hline & Emocional & Físico & Mental \\
\hline Porcentaje & $90 \%$ & $5 \%$ & $5 \%$ \\
& & Total 100\% & \\
\hline
\end{tabular}

En el centro, el trabajador social realiza diferentes actividades para lograr la resiliencia en el adulto mayor. Un 90\% de ellos respondieron que éste les beneficia en estado emocional, un $5 \%$ en el aspecto físico y otro $5 \%$ mental. Por lo que se concluye que la labor del trabajador social es beneficiosa en su totalidad, ya sea en su estado mental, físico y el más importante emocional para una buena resiliencia.

\section{Tabla 7}

¿Cómo interviene el trabajador social del centro para lograr la resiliencia del adulto mayor?

\begin{tabular}{l|ccc}
\hline & $\begin{array}{c}\text { Actividades } \\
\text { lúdicas y } \\
\text { recreativas }\end{array}$ & $\begin{array}{c}\text { Charlas } \\
\text { motivacionales }\end{array}$ & Entrevistas \\
\hline Porcentaje & $52 \%$ & $45 \%$ & $3 \%$ \\
\hline
\end{tabular}

Al enfrentar situaciones difíciles, el adulto mayor tiene la necesidad de superarlas él mismo, es por eso que al preguntar cómo interviene el trabajador social del centro para lograr la resiliencia del adulto mayor, el 52\% respondieron que mediante actividades lúdicas y recreativas, el $45 \%$ mediante charlas motivaciones y el $3 \%$ con entrevistas. Por lo que se concluye que las actividades lúdicas y recreativas es lo que más beneficia a estas personas que se encuentran en su momento en un estado de vulnerabilidad.

\section{Discusión}


Medina (2016), expresa que el abandono del adulto mayor se produce cuando se pierde la comunicación con el familiar, es decir que los sentimientos hacia ellos pasan al olvido lo que provoca el aislamiento desplazando los lazos afectivos ocasionando un quiebre dentro de una familia.

Las entrevistas realizadas indican que son pocos los adultos mayores que tienen un familiar cercano y aun así son aislados del hogar. Muchas son las razones por las que han sido abandonados, entre las más mencionadas por los mismos están que sus hijos ya tienen su propia familia y domicilio y no tienen tiempo ni lugar para ellos, o simplemente no tienen familiares a quienes puedan acudir, de modo que la comunicación se interrumpe cortando toda relación e interacción entre los componentes familiares, lo que conllevan a que el individuo tenga graves afecciones en las emociones, de salud y sentimentales y considere necesario acudir al centro diurno para tener un lugar en donde pasar el día.

En este orden de ideas, según la perspectiva de Grotberg, las fuentes se dividen en tres categorías: aquella que indica que la persona puede recibir apoyo (yo tengo), las que se relacionan con las fortalezas intrapsíquicas y condiciones internas de cada persona (yo soy, yo estoy) y aquella que tiene relación con las habilidades de la persona para relacionarse con los demás y resolver los (yo puedo) (Kotliarenco, 1999). Estas categorías se complementan con lo que indica Saavedra (2003), quien desarrolló un modelo emergente del estudio de casos en donde menciona que la respuesta a la resiliencia es realizar acciones orientadas hacia las metas es una acción orientada a metas, lo que permite abordar deferentes problemas desde una perspectiva positiva, caracterizada por elementos afectivos y cognitivos positivos o proactivos ante los problemas, los cuales tienen como base las creencias y vínculos sociales que impregnan la memoria de seguridad y que de modo recursivo interpreta la acción específica y los resultados.

En este sentido, lo mencionado por Edith Grotberg y Saavedra se relaciona con la investigación realizada, por cuanto los resultados obtenidos en la aplicación del cuestionario con las interrogantes vinculadas a las categorías yo tengo, yo soy, yo estoy y yo puedo, además de los factores como la autonomía, vínculos, generatividad, redes y modelos, demuestran la resiliencia de los adultos mayores del Centro Diurno Gerontológico Cristo Rey frente al abandono de sus familiares. Estos 


\section{2}

resultados reflejan que este grupo de atención prioritaria tiene la necesidad de acudir a un lugar en donde poder pasar el día acompañados de otras personas a pesar de que han sido abandonados por sus familiares, a fin de fortalecer su nivel de resiliencia y por ende lograr un envejecimiento activo.

A su vez, la intervención del Trabajador Social del Centro Diurno Gerontológico Cristo Rey forma parte fundamental en la resiliencia del adulto mayor, debido a que se trabaja exclusivamente con actividades que fortalecen al individuo tanto emocional, física y mentalmente con las actividades lúdicas y recreativas, las cuales se ha convertido en el mayor centro de distracción para los miembros de este centro, permitiéndoles crear convivencia, interacción, lazos amistosos y por ende, pueden desarrollar la función de la superación de las adversidades, lo que se corrobora por lo expuesto por Lázaro y Juárez (2015). En este sentido, en el Centro Gerontológico Cristo Rey se determinó que una de las principales funciones de un Trabajador Social es facilitar que todos los adultos mayores desarrollen plenamente sus potencialidades y de esta manera tengan un envejecimiento activo, enriquezcan sus vidas, y eviten prevenir ciertas disfunciones a causa del abandono de sus familiares, muy a tino lo expuesto por Juárez y Lázaro (2015), cuando considera que los Trabajadores Sociales son agentes de cambio.

\section{Conclusión}

Con el pasar de los años, el tema del adulto mayor ha producido un gran apogeo puesto que los niveles de abandono por parte de sus familiares son muy altos; por ende, los individuos están expuestos a contraer enfermedades y desequilibrios emocionales, para lo cual el Ministerio de Inclusión económica y Social promueve el envejecimiento activo, para lograr una vida digna y saludable a través de los centro de atención directa, entre ellos el Centro Diurno Gerontológico Cristo Rey, en donde el trabajador social junto a su equipo interdisciplinario tienen la ardua labor de lograr este propósito. En este centro se pueden observar adultos mayores con alta resiliencia, ellos indican que acudir a este lugar les llena de vida y que la mejor manera de superar las situaciones difíciles por las que pasan es ser muy optimistas, tener una buena relación con sus compañeros, formar lazos fraternos y lo más importante participar en las actividades lúdicas y recreativas que día a día se realizan 
en el centro con la dirección de la trabajadora social y su equipo interdisciplinario. Es ahí donde el adulto mayor encuentra la familia que hace mucho tiempo desapareció.

\section{Referencias}

Brandtstädter, y Greve. (1994). resiliencia y vejez. Portal de mayores.

Cárdenas, y Cedeño. (2018). Intervención del trabajo social en los programas de atención al adulto mayor en los centros geriátricos en la ciudad de Portoviejo. Revista Caribeña de Ciencias Sociales.

Cárdenas, Jiménez, López, Díaz, y Alba. (2011). Resiliencia en la vejez. Revista de Salud Pública, 3-14.

Casas, y Campos. (2008). Resiliencia, experiencias investigativas y prospectivas. Economía, Organizacion y Ciencias Sociales, 14-204.

Constitución de la República del Ecuador. (2008). Asamblea Nacional Constituyente de Ecuador de 2007-2008. 18-136.

Constitución de la República del Ecuador. (2008). Constitución de la República del Ecuador. Asamblea Nacional Constituyente de Ecuador de 2007-2008.

Ecuador, C. d. (2008). Constitucion de la República del Ecuador. Ecuador: 2008.

Garmezy. (2006). Resiliencia: definición, características y utilidad del concepto. Revista de Psicopatología y Psicología Clínica, 3-22.

Instituto Nacional de Estadística y Censos . (12 de Noviembre de 2016). Reconocer el envejecimiento: el primer paso. EL TELÉGRAFO, pág. 1.

Jiménez. (2011). Papel de la resiliencia en personas mayores institucionalizadas. Universidad Pontificia ICAI ICADE Comillas Madrid, 11-32.

Jiménez Ambriz, M. G. (s.f.). xv resiliencia y vejez. Portal de mayores.

Juárez, y Lázaro. (2015). Resiliencia, experiencias investigativas y prospectivas. Obtenido de Economía. organizacion y ciencias sociales: https://www.3ciencias.com/wp-content/uploads/2018/07/Resiliencia.pdf

Juárez, L. y. (2015). Trabajo Social y Resiliencia: Intervenir desde las fortalezas. Universidad Pontificia Comillas de Madrid. Obtenido de http://www10.ujaen.es/sites/default/files/users/factra/Congreso/1 .pdf 
Kotliarenco, M. (1999). Algunas particularidades metodológicas en los estudios sobre Resiliencia. Chile: MAK consultores.

Lascano. (2017). . Estrategias de actuación ante el abandono familiar a los jubilados del dispensario del seguro social campesino Santa Lucía. Bachelor's thesis.

Lázaro, y Juárez. (2015). Trabajo social y resiliencia, intervenir desde las fortalezas. Obtenido de Universidad Pontificia Comillas de Madrid: https://factra.ujaen.es/sites/centro_factra/files/uploads/Congreso/1.pdf

Lázaro, J. y. (2008). Resiliencia, experiencias investigativas y prospectivas. Economia, Organizacion y Cinecias Sociales, 14-204.

Machado, y Nunes, F. (2010). Estudio sobre la resiliencia en ancianas del Valle de Paraíba, São Paulo, Brasil. Revista electronica trimestral de enfermeria, 3-18.

Medina Medina, J. R. (2016). El abandono del adulto mayor y su incidencia a la mendicidad en la ciudad de Quito en el año 2014, frente a la obligación del Estado a brindar protección. Bachelor's thesis, Quito: UCE.

Medina, J. (2016). Proyecto de Investigación. Obtenido de el abandono del adulto mayor y su incidencia a la mendicidad en la ciudad de quito en el año 2014, frente a la obligación del estado a brindar protección: http://www.dspace.uce.edu.ec/bitstream/25000/5817/1/T-UCE-0013-Ab037.pdf

Organización Mundial de la Salud. (2002). Envejecimiento activo: un marco político. Revista Española de Geriatria y gerontología, 6-32.

Organización Mundial de la Salud. (25 de Agosto de 2015). Capítulo 5. Obtenido de El maltrato de las personas mayores: https://www1.paho.org/Spanish/AM/PUB/capitulo_5.pdf

Ramos, Gonzales, y Pacheco. (2015). "Calidad de vida en el adulto mayor". VARONA. Obtenido de http://www.redalyc.org/articulo.oa? id=360643422019 Saavedra, E. (2003 ). El Enfoque Cognitivo Procesal Sistémico, como posibilidad de Intervenir educativamente en la formación de sujetos Resilientes. España: Universidad de Valladolid. 